\title{
Aplicação de regressão baseada no uso do solo para predizer a concentração de material particulado inalável no município de São Paulo, Brasil
}

\section{Application of land use regression to predict the concentration of inhalable particular matter in São Paulo city, Brazil}

\author{
Mateus Habermann \\ Departamento de Medicina Preventiva da Faculdade de Medicina da Universidade de São Paulo (USP) - São Paulo (SP), Brasil. \\ Nelson Gouveia \\ Departamento de Medicina Preventiva da Faculdade de Medicina da USP - São Paulo (SP), Brasil.
}

\begin{abstract}
Resumo
O estudo teve por objetivo construir um modelo de regressão baseada no uso do solo para predizer a concentração material particulado inalável (MP ${ }_{10}$ ) no município de São Paulo, Brasil. O estudo se baseou na média de MP $_{10}$ de 2007 de 9 estações de monitoramento. Obtiveram-se dados demográficos, viários e de uso do solo em círculos concêntricos de 250 a 1.000 m para compor o modelo. Calculou-se regressão linear simples para selecionar as variáveis mais robustas e sem colinearidade. Quatro variáveis entraram no modelo de regressão múltipla. Somente tráfego leve em círculos concêntricos s250 m permaneceu no modelo final, que explicou $63,8 \%$ da variância de $\mathrm{MP}_{10}$. Verificou-se que o método de regressão baseada no uso do solo é rápido, de fácil execução. Entretanto, este modelo se baseou em medições de $\mathrm{MP}_{10}$ de poucos locais.
\end{abstract}

Palavras-chave: poluição do ar; exposição ambiental; material particulado; impacto ambiental; saúde ambiental.

\section{Abstract}

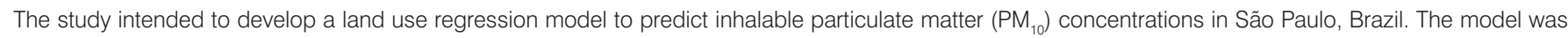
based on the 2007 average of $\mathrm{PM}_{10}$ available from 9 monitoring stations and demographic, roads and land use data in buffers of 250 to 1,000 m. Simple linear regression were used to select the more robust independent variables and those without collinearity. Four variables were selected for the multiple regression analysis. Only light traffic in buffers $\leq 250 \mathrm{~m}$ remained in the final model which explained $63.8 \%$ of the $\mathrm{PM}_{10} \mathrm{variance}$.The land use regression approach is a quick and easy method to predict air pollution levels. However, our model was based on measurements of only a few sites.

Keywords: air pollution; environmental exposure; particulate matter; environmental impact; environmental health.

\section{Introdução}

Exposição é um termo amplo, usado para denotar quaisquer dos atributos de um sujeito ou agente (contaminantes) que pode ser causa ou preditor de um agravo. Ela consiste no contato entre o indivíduo (via inalação, ingestão, contato dérmico) e o contaminante, em diferentes cenários ambientais (ar, água e solo) e envolve o quanto este foi ingerido ou absorvido pelo organismo (ZOU et al., 2009).

A abundância de um poluente, definido como massa ou fração do material por volume ou área no ambiente, aumenta a chance de contato com o contaminante com a população alvo em um dado tempo/espaço. O ambiente pode agir, portanto, como um facilitador da exposição aos poluentes e, com isso, desencadear diversos desfechos (NUCKOLS; WARD; JARUP, 2004).

As avaliações de exposição abordam o grau de interação entre as fontes contaminantes em vários níveis de detalhe, podendo incluir três abordagens de monitoramento: biológico (biomarcadores), pessoal (medições pessoais) e ambiental (modelagem ambiental) (ZOU et al., 2009).

As diferentes abordagens para avaliar exposição foram hierarquizadas pela US National Research Council e as abordagens baseadas 
em informações individuais foram consideradas as melhores formas de estimar exposição real. Em contrapartida, aquelas baseadas em dados residenciais ou ocupacionais em áreas geográficas definidas foram consideradas menos acuradas (NIEUWENHUIJSEN; PAUSTENBACH; DUARTE-DAVIDSON, 2006). No entanto, devido aos altos custos das avaliações individuais, tem-se buscado aperfeiçoar os métodos relacionados a medições e modelagens ambientais, assumindo que essas medições sejam uma boa aproximação da exposição individual nos estudos epidemiológicos.

Alguns estudos avaliam a poluição atmosférica utilizando as concentrações médias dos poluentes medidos pelas estações de monitoramento da qualidade do ar. No entanto, por motivos de caráter econômico e administrativo há limitação no número e na distribuição dessas estações, o implica na mensuração apenas da concentração regional dos poluentes sem detectar a heterogeneidade espacial dos mesmos, que possuem alta variação espacial a poucas distâncias da fonte geradora (SAHSUVAROGLU et al., 2006).

Há locais em que a concentração da poluição atmosférica é mais elevada. As causas para isso podem ser a dispersão limitada de poluentes, como corredores de prédios (street canyons), ou emissões elevadas originárias do tráfego intenso, indústrias, estações ferroviárias, aeroportos e portos (WHO, 2006). Por isso, modelos baseados apenas em medições de estações de monitoramento não refletem com exatidão a exposição à poluição atmosférica de pessoas residentes próximas a estas áreas.

Muitos modelos de exposição ambiental à poluição atmosférica se baseiam na proximidade da fonte poluidora, por exemplo, a proximidade ao tráfego (JERRET; FILKENSTEIN, 2005). Embora esta abordagem seja mais simples, ela é limitada por considerar que os sujeitos estão igualmente expostos e desconsidera outros parâmetros que influenciam a dispersão dos poluentes, por exemplo, topografia, uso do solo, temperatura e estagnação atmosférica (JERRET; FILKENSTEIN, 2005; RYAN; LEMASTERS, 2007). Portanto, esses modelos vêm se apresentando cada vez mais refinados ao longo do tempo, incluindo variáveis que influenciam na dispersão dos poluentes (ZOU et al., 2009) capazes de identificar variações, em pequena escala, dos níveis dos poluentes, como tipo de rodovia/via, fluxo de tráfego e uso do solo, densidade populacional etc.

Um método que se tornou frequente na literatura sobre poluição atmosférica é a regressão baseada no uso do solo (land use regression). Ele estima os níveis de poluição em uma dada área, baseado em características que se atribuem estar relacionadas com a concentração e dispersão dos poluentes (SU et al., 2009) e utiliza medições de poluição em um determinado local como variável dependente e o tipo de uso do solo, tráfego e características geográficas dentro de círculos concêntricos (buffers) ao redor deste local como preditores das concentrações medidas (ARAIN et al., 2007).

Este estudo teve por objetivo construir um modelo de regressão baseado no uso do solo para predizer as concentrações ambientais de $\mathrm{MP}_{10}$ no município de São Paulo.

\section{Metodologia}

O estudo foi realizado no município de São Paulo (MSP), que se constitui como centro de uma grande metrópole, com uma população de 11.324.102 habitantes, alta densidade demográfica $\left(\approx 7.500\right.$ habitantes por $\left.\mathrm{km}^{2}\right)$, numa área de $1.509 \mathrm{~km}^{2}$ e um grau de urbanização de 98,9\% (SÃO PAULO, 2011). A cidade caracteriza-se pela elevada emissão de poluentes, principalmente atribuída à frota veicular superior a 7 milhões de veículos (SÃO PAULO, 2011) circulando em $\approx 16.300 \mathrm{~km}$ de vias.

Dados da média anual de $\mathrm{MP}_{10}$ para o ano de 2007 foram obtidos de medições das estações automáticas de monitoramento da qualidade do ar localizadas no município de São Paulo, e disponibilizadas no sítio eletrônico da Companhia de Tecnologia de Saneamento Ambiental (CETESB), cujas medições eram representativas para o ano em questão. Nesse caso, a adoção de critérios de representatividade dos dados para uma determinada estação ou período significa que as medições não apresentaram falhas que comprometam a interpretação do resultado obtido. No caso da média diária, é representativa a informação que engloba dois terçosdas medições horárias válidas no dia ( $\geq 16$ horas). A média anual é considerada representativa se atingirem pelo menos metade das medições diárias válidas para os quadrimestres janeiro-abril, maio-agosto e setembro-dezembro (SÃO PAULO, 2008).

Foram utilizadas informações do Censo 2000 do Instituto Brasileiro de Geografia e Estatística (IBGE), como total de residentes em domicílios particulares e total de domicílios particulares, por setor censitário. As informações de altitude do município, em intervalos de $5 \mathrm{~m}$, foram obtidas da base de dados Geolog da Prefeitura de São Paulo.

A base cartográfica de uso do solo, por quadra fiscal, do MSP foi elaborada pela Secretaria Municipal de Planejamento (SEMPLA) e conta com 15 classes de predominância de uso, por tipologia de ocupação (horizontal e vertical), padrão das edificações (baixo, médio e alto), tipo de uso (residencial, industrial, comércio e serviços, garagens, escolas, equipamentos de uso público, outros usos), terrenos vagos e sem predominância.

A Companhia de Engenharia de Tráfego (CET) realiza contagem de veículos em algumas vias do município, nos horários de pico da manhã e utilizam o programa canadense EMME-2 para simular o tráfego nas demais vias do município. Baseando-se nas contagens e simulações, a CET forneceu informações do tráfego médio de 2007. Também foram incluídos dados das vias, por tipo (vias locais, coletoras, arteriais e de trânsito rápido), número de faixas de rolamento e velocidade média do fluxo.

Todas as variáveis independentes de uso do solo, demográficas, tráfego e altitude foram construídas utilizando o programa de sistema de informação geográfica (SIG) Mapinfo (Professional version 8.5; Mapinfo Corporation, New York, NY, USA) e se baseou em círculos concêntricos de 250, 500, 750 e $1.000 \mathrm{~m}$ de raio no entorno das estações de monitoramento da CETESB (Figura 1). Foi possível obter 
um total de 113 variáveis independentes exploradas na construção do modelo final e são apresentadas de forma resumida no Quadro 1.

Foi criado um mapa de 6.098 pontos regulares de 500x500 m do MSP, no qual se aplicou a fórmula obtida pelo modelo de regressão final, de forma a predizer os níveis de $\mathrm{MP}_{10}$ para cada ponto. O mapa da concentração de $\mathrm{MP}_{10}$ no município em 2007 (em $\mu \mathrm{g} . \mathrm{m}^{3}$ ) foi elaborado no SIG ArcGIS (versão 9.3.1 ESRI, Redlands, CA, USA) por meio da ferramenta de interpolação, que se baseou no método denominado Kriging, atribuindo valores da variável de interesse em pontos desconhecidos utilizando valores de pontos conhecidos.

A associação entre características de uso do solo, demográficas, tráfego e altitude obtidas pelas 113 variáveis independentes com os níveis de $\mathrm{MP}_{10}$ da respectiva estação de monitoramento foi avaliada por regressão linear simples ou univariada (erro tipo I ou $\alpha=5 \%$ ). Aquelas com valor preditivo (valor $\mathrm{p}$ ) $\leq 0,2$ nesta análise foram selecionadas para cálculo do modelo final. A colinearidade das variáveis foi examinada pelo teste de correlação de Pearson. No caso de haver colinearidade, foi selecionada aquela mais robusta (menor valor $\mathrm{p}$ ). Esses cálculos foram realizados no programa SPSS para Windows, versão 13.0.

O modelo final foi obtido por regressão linear múltipla, pelo método bootstrap (MOORE et al., 2007), no qual as análises foram replicadas 150 vezes. As análises múltiplas eram refeitas, com a exclusão das variáveis sem significância estatística. Esta prática foi repetida até se obter $a(s)$ variável(s) com $p \leq 0,05$. A regressão linear múltipla foi realizada no programa Statistics Data Analysis 9.0 (STATA).

Por fim, a fórmula obtida na regressão múltipla foi aplicada no mapa de pontos 500x500 m do MSP para o mapeamento por interpolação.

\section{Resultados}

Das 12 estações de monitoramento da CETESB que mediram a concentração de $\mathrm{MP}_{10}$, em 2007, somente 9 foram representativas e utilizadas para construção do modelo. A concentração média de $\mathrm{MP}_{10}$ obtida dessas medições das estações de monitoramento foi de 40,1 ug.m ${ }^{-3}$ (desvio padrão $\left.[\mathrm{DP}]=4,6 \mathrm{ug} \cdot \mathrm{m}^{-3}\right)$. As estações de monitoramento Cambuci $\mathrm{e}$ Congonhas foram as que apresentaram maior média de $\mathrm{MP}_{10}$, seguida pela estação Centro (Tabela 1).

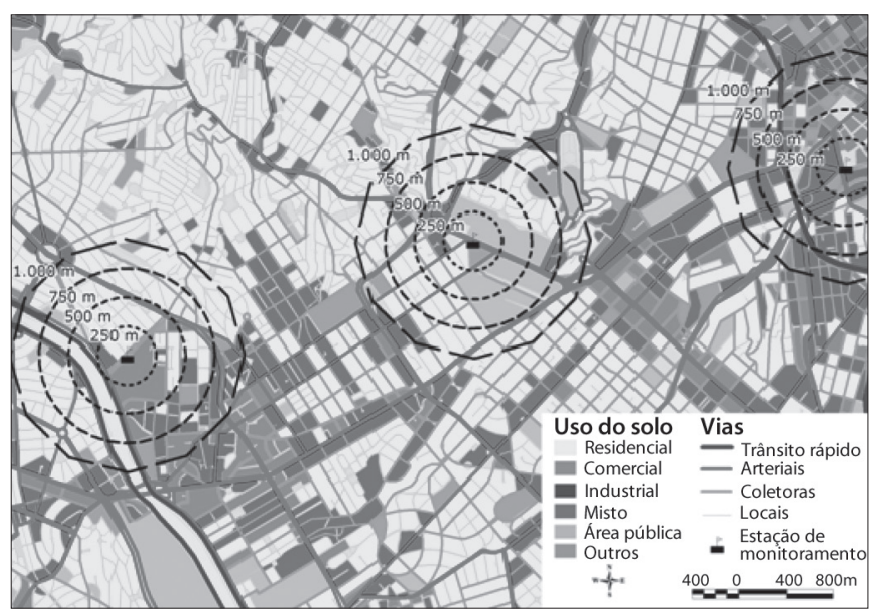

Figura 1 - Representação das estações de monitoramento da CETESB e dos círculos concêntricos construídos para obtenção das variáveis do modelo de regressão baseada no uso do solo.

Quadro 1 - Variáveis utilizadas para construção do modelo de regressão baseada no uso do solo no município de São Paulo.

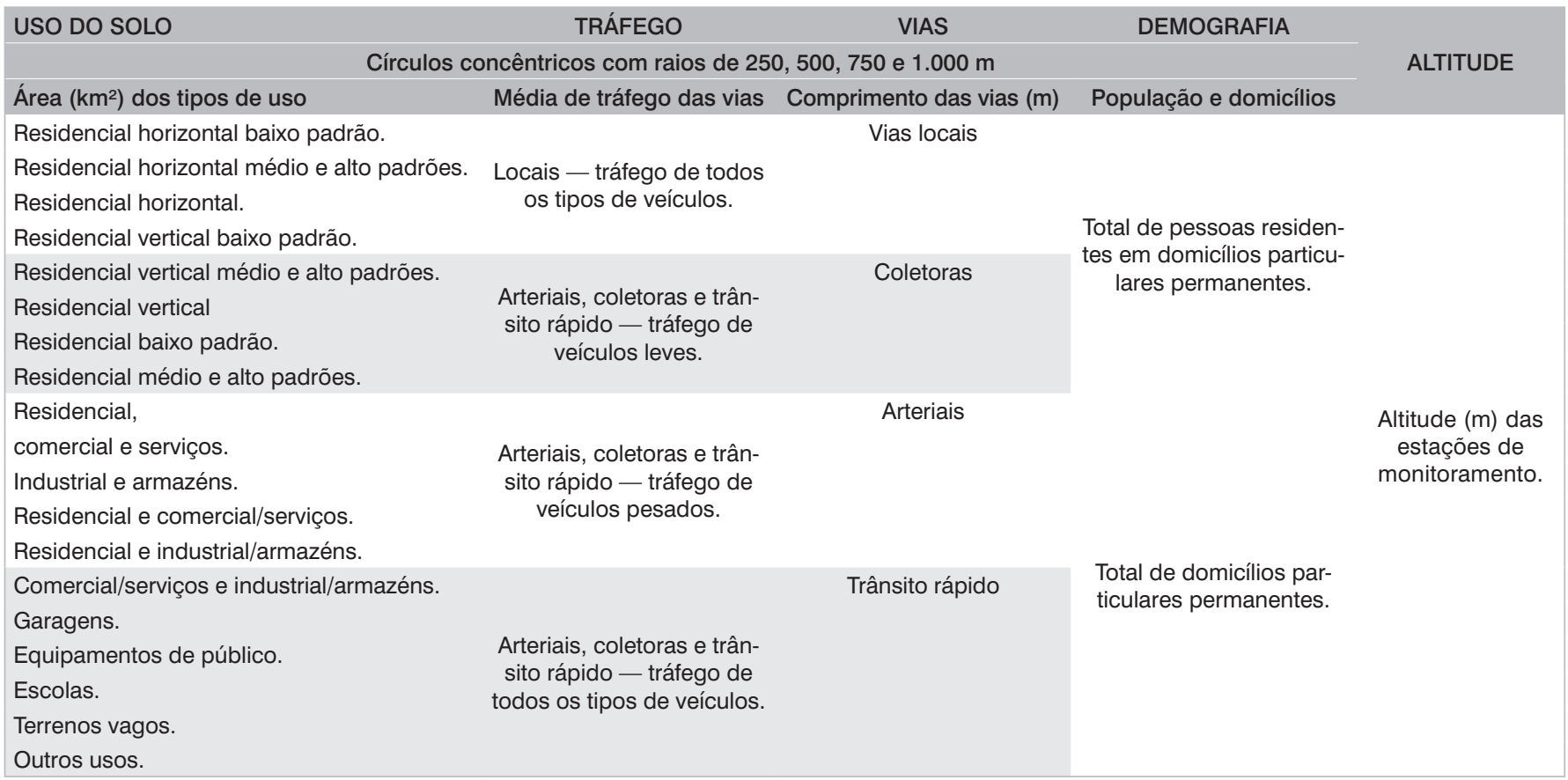


Do total de 113 variáveis construídas por SIG, 24 delas apresentaram $\mathrm{p} \leq 0,2$ na análise de regressão linear univariada. Estas se referiram ao tráfego de veículos, extensão de vias, total de domicílios, uso residencial, terrenos vagos e indústrias/armazéns dos círculos concêntricos. No entanto, 20 delas apresentaram colinearidade e foram excluídas da análise, restando apenas quatro para o cálculo de regressão multivariada. Três variáveis finais foram associadas ao aumento dos níveis de material particulado (coeficiente ou $\beta$ positivo): tráfego de veículos leves; total de domicílios; uso comercial/serviços e industrial/armazéns. Inversamente, o uso residencial horizontal de médio e alto padrões foi negativamente associado com o nível de material particulado (Tabela 2).

Tabela 1 - Média anual de MP ${ }_{10}$ das estações de monitoramento do município de São Paulo, 2007.

\begin{tabular}{|c|c|}
\hline Estação de monitoramento & média de $\mathrm{MP}_{10}\left(\mathrm{em}\right.$ ug. $\left.\mathrm{m}^{-3}\right)$ \\
\hline Cambuci & 46 \\
\hline Congonhas & 46 \\
\hline Centro & 45 \\
\hline Parque Dom Pedro II & 41 \\
\hline Cerqueira César & 39 \\
\hline Ibirapuera & 38 \\
\hline Nossa Senhora do Ó & 36 \\
\hline Santo Amaro & 36 \\
\hline Pinheiros & 34 \\
\hline Mooca & $s / r$ \\
\hline Parelheiros & $s / r$ \\
\hline Santana & $s / r$ \\
\hline
\end{tabular}

s/r: sem representatividade
Apenas a média diária do tráfego de veículos leves das vias coletoras, arteriais e de trânsito rápido até $250 \mathrm{~m}$ apresentou-se estatisticamente significante $(p<0,001)$ e permaneceu no modelo de regressão multivariada, que reportou $\mathrm{r}^{2}=0,638$, conforme se observa na Tabela 3 .

No mapa de pontos regulares $500 \times 500 \mathrm{~m}$ se aplicou a fórmula da regressão para predizer o valor de $\mathrm{MP}_{10}$ em 2007 $\left(\mathrm{PM}_{10} 2007=35.31484+(0,0005114 *\right.$ traf_auto250 $)$. A média predita de $\mathrm{MP}_{10}$ pelo modelo de regressão foi de 36,35 ug.m $\mathrm{m}^{-3}$ $\left(\mathrm{DP}=3,24 \mathrm{ug} \cdot \mathrm{m}^{-3}\right)$. A interpolação desses pontos gerou o mapa da concentração média diária deste poluente no município de São Paulo em 2007.

Conforme se observa na Figura 2, a concentração de $\mathrm{MP}_{10}$ é maior ao longo de vias com tráfego elevado e nos distritos centrais, onde há maior tráfego. Em contrapartida, as concentrações diminuem nas partes periféricas, principalmente no extremo sul do município de São Paulo.

\section{Discussão}

O modelo de regressão final do $\mathrm{MP}_{10}$ desenvolvido neste estudo incluiu apenas uma variável independente (média diária do tráfego

Tabela 3 - Resultados do modelo final da regressão linear multivariada.

\begin{tabular}{lcccc} 
Variável & $\begin{array}{c}\text { Coeficiente } \\
(\beta)\end{array}$ & $\begin{array}{c}\text { Erro padrão } \\
\left(\hat{\mathbf{S}}_{\text {boot }}\right)\end{array}$ & $\mathbf{z}$ & Valor $\mathrm{p}$ \\
\hline Constante & 35,31484 & 1,394 & 25,34 & $<0,001$ \\
$\begin{array}{l}\text { Tráfego de veícu- } \\
\text { los leves } \leq 250\end{array}$ & $5,114 \times 10^{-4}$ & $1,33 \times 10^{-4}$ & 3,85 & $<0,001$ \\
$\mathrm{r}^{2}=0,6384$ & & & &
\end{tabular}

Tabela 2 - Variáveis finais do modelo univariado $(p \leq 0,2)$.

\begin{tabular}{|c|c|c|c|c|c|}
\hline Variável & Buffer (m) & $r$ & $r^{2}$ & Coeficiente $(\beta)$ & Valor $p$ \\
\hline Tráfego de veículos leves & 250 & 0,799 & 0,638 & $1,0 \times 10^{-3}$ & 0,01 \\
\hline Tráfego de veículos* & 250 & 0,727 & 0,528 & 0 & 0,027 \\
\hline Extensão de vias arteriais* & 250 & 0,717 & 0,514 & $3,0 \times 10^{-3}$ & 0,03 \\
\hline Extensão de vias arteriais* & 500 & 0,64 & 0,41 & $1,0 \times 10^{-3}$ & 0,063 \\
\hline Uso residencial horizontal* & 500 & 0,635 & 0,403 & $-2,35 \times 10^{-5}$ & 0,066 \\
\hline Uso residencial horizontal — médio e alto padrões* & 750 & 0,63 & 0,397 & $-1,17 \times 10^{-5}$ & 0,069 \\
\hline Uso residencial horizontal* & 750 & 0,63 & 0,397 & $-1,10 \times 10^{-5}$ & 0,069 \\
\hline Uso residencial horizontal — médio e alto padrões* & 500 & 0,628 & 0,395 & $-2,45 \times 10^{-5}$ & 0,07 \\
\hline Extensão de vias coletoras* & 1.000 & 0,579 & 0,335 & 0 & 0,102 \\
\hline Total de domicílios particulares permanentes & 250 & 0,573 & 0,329 & $4,0 \times 10^{-3}$ & 0,107 \\
\hline Extensão de vias arteriais* & 1000 & 0,571 & 0,326 & 0 & 0,108 \\
\hline Extensão de vias arteriais* & 750 & 0,555 & 0,308 & $1,0 \times 10^{-3}$ & 0,121 \\
\hline Uso residencial* & 250 & 0,632 & 0,399 & $-9,62 \times 10^{-5}$ & 0,128 \\
\hline Total de habitantes* & 250 & 0,537 & 0,288 & $2,0 \times 10^{-3}$ & 0,136 \\
\hline Extensão de vias locais* & 1.000 & 0,508 & 0,258 & 0 & 0,163 \\
\hline Uso residencial horizontal — médio e alto padrões & 250 & 0,496 & 0,246 & $-5,56 \times 10^{-5}$ & 0,174 \\
\hline Uso residencial horizontal* & 250 & 0,497 & 0,247 & $-5,55 \times 10^{-5}$ & 0,174 \\
\hline Uso comercial/serviços e industrial/armazéns & 500 & 0,493 & 0,243 & $8,16 \times 10^{-5}$ & 0,177 \\
\hline Total de domicílios particulares permanentes* & 500 & 0,479 & 0,23 & $1,0 \times 10^{-3}$ & 0,192 \\
\hline Uso Industrial e armazéns* & 250 & 0,478 & 0,228 & $1,0 \times 10^{-3}$ & 0,193 \\
\hline Terrenos vagos* & 250 & 0,478 & 0,228 & 0 & 0,193 \\
\hline Extensão de vias locais* & 750 & 0,473 & 0,224 & 0 & 0,198 \\
\hline Extensão de vias coletoras* & 750 & 0,468 & 0,219 & $1,0 \times 10^{-3}$ & 0,2 \\
\hline Tráfego de veículos leves* & 500 & 0,471 & 0,221 & $6,16 \times 10^{-5}$ & 0,2 \\
\hline
\end{tabular}


de veículos leves das vias coletoras, arteriais e de trânsito rápido até $250 \mathrm{~m}$ ). Em geral, a literatura sobre este tema tem reportado modelos finais que incluem diversas variáveis, mesmo assim o modelo foi capaz de explicar 63,8\% da variabilidade na concentração média anual de $\mathrm{MP}_{10}$ no município de São Paulo.

Este percentual está de acordo com o Relatório Anual da Qualidade do Ar de 2007, da CETESB, no qual se estima que 40\% da concentração de $\mathrm{MP}_{10}$ na região metropolitana de São Paulo é originária da queima de combustível veicular e os outros 25\% da ressuspensão de partículas (não detalhando se é ocasionado pelo tráfego), que somados totalizam 65\% (SÃO PAULO, 2008).

Alguns autores relataram o uso de dados apenas da rede de monitoramento da qualidade do ar para gerar modelos de regressão baseada no uso do solo. Este foi o caso do modelo de Moore et al. (2007)

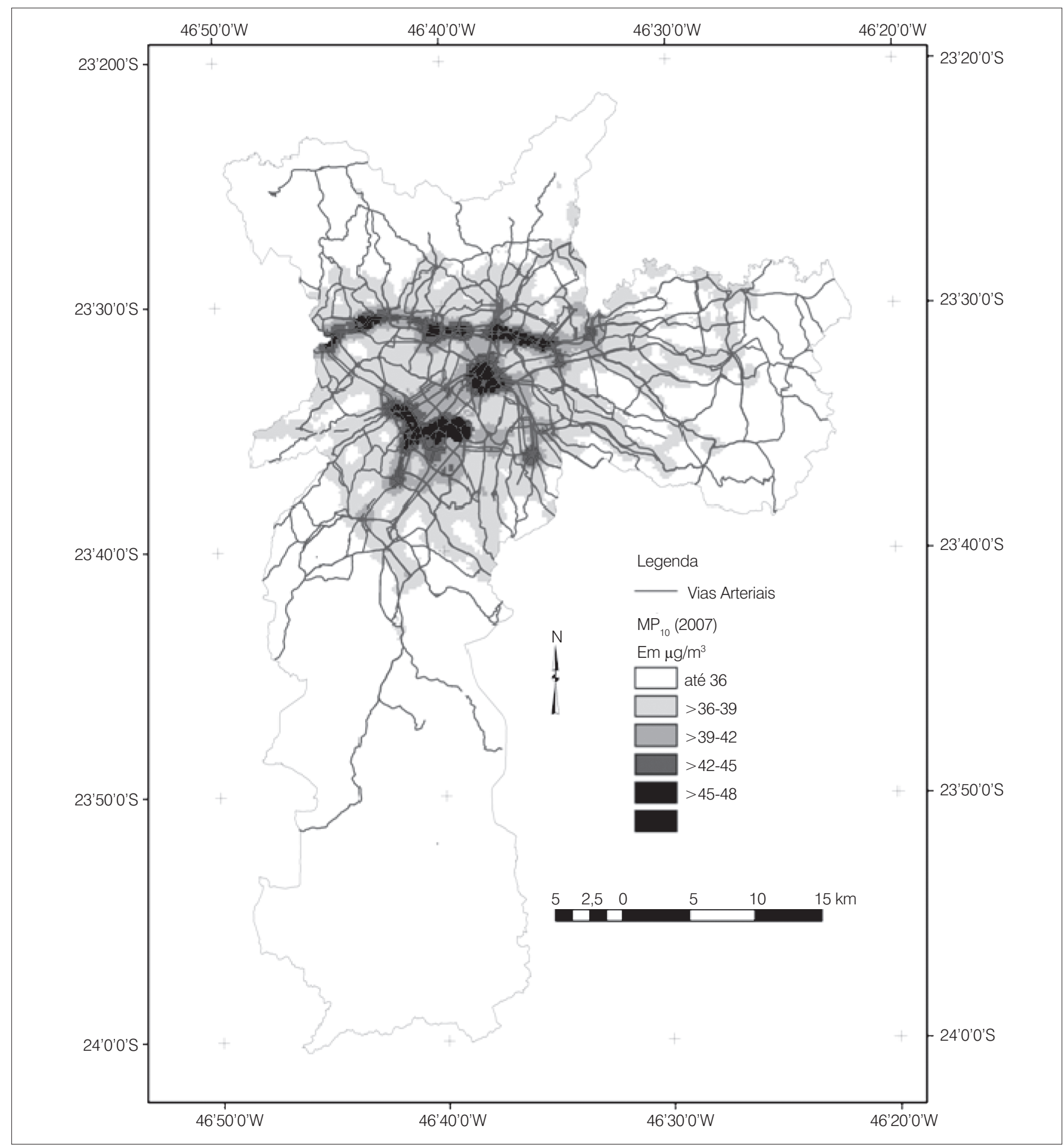

Figura 2 - Média anual da concentração de $\mathrm{MP}_{10}\left(\mathrm{em} \mu \mathrm{g} / \mathrm{m}^{3}\right)$ no município de São Paulo, 2007. 
para predizer $\mathrm{PM}_{2,5}$ na região metropolitana de Los Angeles (EUA), baseado em dados de 23 estações de monitoramento. O modelo finalapresentou $r^{2}=0,63$ e se mantiveram as variáveis relativas a tráfego e áreas governamentais e industriais. O modelo foi aplicado em pontos aleatórios e os valores estimados foram mapeados por interpolação.

O outro foi o estudo desenvolvido em Shizuoka, Japão (KASHIMA et al., 2009) e utilizou dados de medições da rede de monitoramento local. $\mathrm{O}$ modelo final de $\mathrm{NO}_{2}$ apresentou poder preditivo de $52 \%$ e incluiu dados de densidade de vias, tráfego, distância do litoral e terreno cultivável. O modelo de partículas suspensas totais incluiu somente volume de tráfego de caminhões e o seu poder preditivo foi baixo $\left(r^{2}=0,11\right)$.

Apesar da possibilidade de utilizar dados de estações de monitoramento, a literatura aborda o maciço uso de amostradores (samplers) para coleta de informações de poluição atmosférica. As medições são realizadas ao longo das áreas onde se pretende desenvolver modelos de regressão para estimar a concentração de poluentes.

Gilbert et al. (2009) mediram $\mathrm{NO}_{2}$ em Montreal/Canadá por meio de 67 amostradores. O modelo final predisse 54\% da variação deste poluente e incluiu o comprimento de vias movimentadas, fluxo veicular, uso de solo industrial e densidade populacional.

Ross et al. (2006) mediram $\mathrm{NO}_{2}$ com 39 amostradores em San Diego (EUA). O modelo final $\left(\mathrm{r}^{2}=0,79\right)$ foi aplicado em 500 pontos aleatórios e foi feita interpolação para mapear a dispersão de $\mathrm{NO}_{2}$ na área estudada.

O modelo desenvolvido em Roma por Rosenlund et al. (2009) se baseou em 70 locais de medição de $\mathrm{NO}_{2}$. O poder preditivo do modelo foi de $68 \%$ e incluiu a distância de vias movimentadas, tamanho do setor censitário, número de residentes, densidade populacional e altitude.

Brauer et al. (2003) desenvolveram um modelo de $\mathrm{PM}_{2,5}$ na Holanda, Munique (Alemanha) e Estocolmo (Suécia), utilizando entre 40 e 42 amostradores em cada localidade. Os modelos finais explicaram 73, 56 e 50\% da variabilidade média anual de $\mathrm{PM}_{2,5}$ na Holanda, Munique e Estocolmo, respectivamente.

Su et al. (2009) modelaram concentrações de $\mathrm{NO}, \mathrm{NO}_{\mathrm{x}}$ e $\mathrm{NO}_{2}$ em Los Angeles (EUA) com dados de medições de 181 amostradores. Os modelos finais explicaram 81, 86 e $88 \%$ da variação de $\mathrm{NO}, \mathrm{NO}_{2}$ e $\mathrm{NO}_{\mathrm{x}}$, respectivamente.

Brauer et al.(2008) estimaram fumaça preta, $\mathrm{MP}_{2,5}, \mathrm{NO}$ e $\mathrm{NO}_{2} \mathrm{em}$ Vancouver (Canadá). O poder preditivo do modelo de $\mathrm{NO}$ ficou em $62,56 \%$ para $\mathrm{NO}_{2}$ e fumaça preta e, por fim, um poder preditivo de $52 \%$ no modelo de $\mathrm{MP}_{2,5}$

Em Valência, Espanha, Iñiguez et al. (2009) desenvolveram um modelo de $\mathrm{NO}_{2}$ com medições de 93 amostradores. O poder preditivo do modelo foi de $81 \%$ e houve tendência de decréscimo dos níveis de $\mathrm{NO}_{2}$ da zona urbana para a rural e grande influência de uma rodovia que cruza a região.

Apenas alguns estudos utilizaram variáveis meteorológicas na construção dos modelos de regressão baseada no uso do solo (ARAIN et al., 2007; BRIGGS et al., 2000; MAUKO; TANG; GEORGE, 2008).
Assim como na regressão baseada no uso do solo desenvolvida no município de São Paulo, a grande maioria dos trabalhos verificados desconsidera a influência da direção/velocidade dos ventos na dispersão dos poluentes ou a sazonalidade dos níveis de poluição, o que pode diminuir a capacidade de predição dos modelos.

Briggs et al., (2000) aplicaram um modelo de $\mathrm{NO}_{2}$ em quatro cidades do Reino Unido, identificando características como temperatura, velocidade do vento e pressão atmosférica. Mauko, Tang e George (2008) construíram dois modelos: com dados de círculos concêntricos e outro com círculos distorcidos para a direção predominante dos ventos. No modelo de círculos concêntricos o $\mathrm{r}^{2}$ foi 0,66 e no modelo baseado em círculos distorcidos o poder preditivo aumentou para 0,81.

No modelo desenvolvido em Toronto e Hamilton (Canadá) (ARAIN et al., 2007), a inclusão de dados eólicos aumentou a predição do modelo de 65 para 69\%. Em outro modelo de Hamilton (SAHSUVAROGLU et al., 2006) com informações de direção do vento o $\mathrm{r}^{2}$ foi de 0,76 .

Embora a literatura não relate a confiabilidade e qualidade das informações de uso do solo, a forma de organização dos dados de uso do solo em São Paulo pode ter limitado o modelo, pois a informação é disponível em categorias prontas e agregadas por quadras, o que impossibilita o aumento do nível de detalhamento, por exemplo, por lotes. Além disso, a dinâmica da cidade faz com que o uso do solo se altere rapidamente e desordenadamente, principalmente nas periferias ou em antigas áreas industriais. Apesar disso, houve associação negativa entre o uso residencial horizontal de médio e alto padrões e $\mathrm{MP}_{10}$ no cálculo do modelo final. Este tipo de uso geralmente se caracteriza pela baixa densidade construtiva, menos domicílios e habitantes por quilometro quadrado, fatores que podem contribuir com menor circulação de veículos.

Os modelos verificados na literatura, em sua maioria, estimaram a concentração de $\mathrm{NO}_{2}$. Ele é considerado um bom indicador da poluição gerada por tráfego veicular e é fácil de medir (ROSE et al., 2009). No caso de São Paulo, havia apenas cinco estações de monitoramento cujos dados de $\mathrm{NO}_{2}$ eram representativos em 2007, enquanto dados de $\mathrm{MP}_{10}$ eram representativos em nove estações.

A maior parte desses estudos apresentou poder preditivo acima de 50\%. Mesmo obtendo um percentual preditivo satisfatório, os resultados do modelo aplicado em São Paulo devem ser observados com precaução, uma vez que este se baseou em medições de poucas estações de monitoramento da qualidade do ar. Os modelos de regressão baseada no uso do solo relatados se basearam em mais pontos, mesmo aqueles que utilizaram medições de estações de monitoramento, variando entre 20 (BRIGGS et al., 2000) a 181 locais (SU et al., 2009) e isso aumenta a precisão dos resultados obtidos.

A aplicação de regressão baseada no uso do solo permite a mensuração da contribuição das diversas fontes nos níveis de poluição do ar (fontes pontuais ou móveis) e isso pode auxiliar para que medidas de controle e combate à emissão de poluentes sejam tomadas. 
A única variável que permaneceu estatisticamente significante no modelo final foi o volume de tráfego de veículos leves até $250 \mathrm{~m}$ da estação de monitoramento, o que indica a influência do tráfego veicular nos níveis ambientais de material particulado em São Paulo. A associação com tráfego veicular está de acordo com outros estudos de regressão baseada no uso do solo aplicados com outros poluentes atmosféricos e que enfatizam a contribuição das fontes móveis nos níveis de poluição atmosférica urbana (ARAIN et al., 2007; BRAUER et al., 2003; GILBERT et al., 2009; KASHIMA et al., 2009; MAUKO; TANG; GEORGE, 2008; ROSENLUND et al., 2009; SAHSUVAROGLU et al., 2006; SU et al., 2009).

Conforme se verificou no mapa de interpolação, as maiores concentrações de $\mathrm{MP}_{10}$ foram em locais de elevado fluxo veicular associado a áreas com maiores densidades populacionais. Isto evidencia o potencial impacto que a exposição ao $\mathrm{MP}_{10}$ pode acarretar à saúde, uma vez que as maiores concentrações atingem um número maior de pessoas.

\section{Considerações Finais}

O modelo de regressão baseada no uso do solo desenvolvido no município de São Paulo foi capaz de explicar mais de 60\% da variabilidade espacial da concentração média de $\mathrm{MP}_{10}$ no ano de 2007. Este poder preditivo está de acordo com estudos similares. A permanência da variável volume de tráfego de veículos leves até $250 \mathrm{~m}$ no modelo final destaca à importância da fonte veicular como contribuinte dos níveis de $\mathrm{MP}_{10}$ no município de São Paulo.

$\mathrm{O}$ pequeno número de pontos de medição de $\mathrm{MP}_{10}$ limita a exatidão deste modelo de regressão baseada no uso do solo. Por isso, recomenda-se a construção de um modelo baseado na medição de $\mathrm{MP}_{10}$ em diversos pontos da cidade por meio de amostradores, de forma a aumentar sua precisão. Também é importante que as informações de uso do solo estejam mais detalhadas.

Deve-se enfatizar que este estudo mostrou como é rápido e fácil desenvolver modelos de regressão de uso do solo para estimar exposição aos poluentes atmosféricos, quando há disponibilidade de dados que envolvem sua elaboração. Os pontos aleatórios criados para aplicação de interpolação poderiam ser substituídos por endereço de sujeitos, de forma a estimar a exposição dos mesmos em estudos epidemiológicos, como verificado em Brauer et al. (2008) e Iñiguez et al. (2009). Tendo em vista que a realização de medições pessoais em estudos epidemiológicos é cara e logisticamente difícil, destaca-se a importância de desenvolver métodos indiretos de avaliação da exposição, entre eles a regressão baseada no uso do solo.

\section{Referências}

ARAIN, M.A.; BLAIR, R.; FINKELSTEIN, N.; BROOK, J.R.; SAHSUVAROGLU, T.; BECKERMAN. B.; ZHANG. L.; JERRET. M. (2007) The use of wind fields in a land use regression model to predict air pollution concentrations for health exposure studies. Atmospheric Environment, v. 41, p. 3453-3464.

BRAUER, M.; HOEK, G.; VLIET, P.; MELIEFSTE, K.; FISCHER, P.; GEHRING, U.; HEINRICH, J.; CYRYS, J.; BELLANDER, T.; LEWNE, M.; BRUNEKREEF, B. (2003) Estimating long-term average particulate air pollution concentrations: application of traffic indicators and geographic information systems. Epidemiology, v. 14, n. 2, p. 228-239.

BRAUER, M.; LENCAR, C.; TAMBURIC, L.; KOEHOORN, M., DEMERS, P.; KARR, C. (2008) A cohort study of traffic-related air pollution impacts on birth outcomes. Environmental Health Perspectives, v. 116, n. 5, p. 680-686.

BRIGGS, D.J.; HOOGH, C.; GULLIVER, J.; WILLS, J.; ELLIOT, P.; KINGHAM, S.; SMALLBONE, K. (2000) A regression-based method for mapping traffic-related air pollution: application and testing in four contrasting urban environments. Science of the Total Environment, v. 253, p. 151-167.

GILBERT, N.L.; GOLDBERG, M.S.; BECKERMAN, B.; BROOK, J.R.; JERRET, M. (2009) Assessing spatial variability of ambient nitrogen dioxide in Montréal, Canada, with a land-use regression model. Air \& Waste Management Association, v. 55, p. 1059-1063.
INIIGUEZ, C.; BALLESTER, F.; ESTARLICH, M.; LLOP, S.; FERNANDEZPATIER, R.; AGUIRRE-ALFARO, A.; ESPLUGUES, A. (2009) Estimation of personal $\mathrm{NO}_{2}$ exposure in a cohort of pregnant women. Science of the Total Environment, v. 407, p. 6093-6099.

JERRET, M.; FINKELSTEIN, M. (2005) Geographies of risk in studies linking chronic air pollution exposure to health outcomes. Journal of Toxicology and Environmental Health, v. 68, p. 1207-1242.

KASHIMA, S.; YORIFUJI, T.; TSUDA, T.; DOI, H. (2009) Application of land use regression to regulatory air quality data in Japan. Science of the Total Environment, v. 407, p. 3055-3062.

MAUKO, M.E.; TANG, B.; GEORGE, L.A. (2008) A sub-neighborhood scale land use regression model for predicting NO2. Science of the Total Environment, v. 398, p. 68-75.

MOORE, D.K.; JERRET, M.; MACK. W.J.; KÜNZLI, N. (2007) A land use regression model for predicting ambient fine particulate matter across Los Angeles, CA. Journal of Environmental Monitoring, v. 9, p. 246-252.

NIEUWENHUIJSEN, M.; PAUSTENBACH, D.; DUARTE-DAVIDSON, R. (2006) New developments in exposure assessment: the impact on the practice of health risk assessment and epidemiological studies. Environmental Health, v. 32, p. 996-1009. 
NUCKOLS, J.R.; WARD, M.H.; JARUP, L. (2004) Using geographic information systems for exposure assessment in Environmental Epidemiology studies. Environmental Health Perspectives, v. 112, n. 9, p. 1007-1015.

ROSE, N.; COWIE, C.; GILLET, R.; MARKS, G.B. (2009) Weighted road density: a simple way of assigning traffic-related air pollution exposure. Atmospheric Environment, v. 43, p. 5009-5014.

ROSENLUND, M.; FORASTIERE, F.; PORTA, D.; SARIO, D.; BADALONI, C.; PERUCCI, C.A. (2009) Traffic-related air pollution in relation to respiratory symptoms, allergic sensitisation and lung function in schoolchildren. Thorax, v. 64, p. 573-580.

ROSS, Z.; ENGLISH, P.B.; SCALF, R.; GUNIER, R.; SMOROKINSKY, S.; WALL, S.; JERRET, M. (2006) Nitrogen dioxide prediction in Southern California using land use regression modeling: potential for environmental health analyses. Journal of Exposure Science and Environmental Epidemiology, v. 16, p. 106-114.

RYAN, P.H.; LEMASTERS, G.K.A (2009) review of land-use regression models for characterizing intraurban air pollution exposure. Inhalation Toxicology, v. 19, n. Suppl.1, p. 127-133.

SAHSUVAROGLU, T.; ARAIN, A.; KANAROGLOU, P.; FINKELSTEIN, N.; NEWBOLD, B. (2006) A land use regression model for predicting ambient concentrations of nitrogen dioxide in Hamilton, Ontario. Air \& Waste Management Association, v. 56, p. 1059-1069.
SÃO PAULO (estado). Companhia de Tecnologia e Saneamento Ambiental (CETESB). (2008) Relatório de qualidade do ar no estado de São Paulo 2007. São Paulo: CETESB, 2008. Disponível em: <http://uww.cetesb.sp.gov.br/ar/ qualidade-do-ar/31-publicacoes-e-relatorios > . Acesso em: ago. de 2009.

SÃO PAULO (estado). Departamento Estadual de Trânsito de São Paulo (DETRAN-SP). (2011) Frota de Veículos - DETRAN-SP. São Paulo: 2011. Disponível em: <http://www.detran.sp.gov.br/>. Acesso em: ago. de 2011.

SÃO PAULO (estado). Secretaria de Planejamento e Desenvolvimento Regional. Fundação Sistema Estadual de Análise de Dados (SEADE). (2011) Município de São Paulo - MSP [internet]. São Paulo: 2011. Disponível em: <http://www.seade.sp.gov.br/produtos/msp/index. php>. Acesso em: ago. de 2011.

SU, J.G.; JERRET, M.; BECKERMAN, B.; WILHELM, M.; GHOSH, J.K.; RITZ, B. (2009) Predicting traffic-related air pollution in Los Angeles using a distance decay regression selection strategy. Environmental Health, v.109, p. 657-670.

WORLD HEALTH ORGANIZATION (WHO). (2006) WHO air quality guidelines global update 2005 - Report on a working group meeting. Bonn, Germany.

ZOU, B.; WILSON, J.G.; ZHAN, F.B.; ZENG, Y. (2009) Air pollution exposure assessment methods utilized in epidemiological studies. Journal of Environmental Monitoring, v. 11, p. 475-490. 